\title{
FINITE TRANSFERABLE LATTICES ARE SHARPLY TRANSFERABLE
}

\author{
C. R. PLATT ${ }^{1}$
}

\begin{abstract}
A lattice $\mathfrak{L}$ is called transferable if and only if, whenever $\mathfrak{E}$ can be embedded into the lattice $I(\mathcal{K})$ of all ideals of a lattice $\mathcal{K}, \mathcal{L}$ can be embedded into $\mathcal{K}$ itself. If for every lattice embedding $f$ of $\mathcal{L}$ into $I(\mathcal{K})$ there exists an embedding $g$ of $\mathcal{L}$ into $\mathcal{K}$ satisfying the further condition that for $x$ and $y$ in $L, f(x) \in g(y)$ holds if and only if $x<y$, then $\mathcal{L}$ is called sharply transferable. It is shown that every finite transferable lattice is sharply transferable.
\end{abstract}

Introduction. A lattice $\mathcal{E}$ is called transferable if and only if, whenever $\mathcal{L}$ can be embedded into the lattice $I(\mathcal{K})$ of all ideals of a lattice $\mathcal{K}, \mathcal{E}$ can be embedded into $\mathcal{K}$ itself. If for every lattice embedding $f$ of $\mathcal{L}$ into $I(\mathcal{K})$, there exists an embedding $g$ of $\mathcal{L}$ into $\mathcal{K}$ satisfying the further condition that for $x$ and $y$ in $L, f(x) \in g(y)$ holds if and only if $x<y$, then $\mathcal{L}$ is called sharply transferable.

The concept of a transferable lattice was introduced in [5], where it was called Property (P). Sharp transferability was later introduced as a related property whose characterization might be more tractable and might shed light on transferability itself. In [3] finite sharply transferable lattices were characterized and in [4] they were shown to be the same as finite sublattices of free lattices. This paper shows that the finite transferable lattices coincide with the finite sharply transferable lattices, thus answering the question raised in [5].

To fix the notation, we recall here some of the definitions from [7]. If $X$ and $Y$ are subsets of a poset, we say that $Y$ dominates $X$ iff for every $x \in X$ there exists $y \in Y$ such that $x \leqslant y$. This is here denoted $X \ll Y$. If $\varrho$ is a lattice, $x \in L$, and $U$

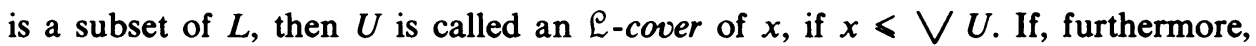
$x \nless u$ for each $u \in U$, then $U$ is a nontrivial $\mathcal{L}$-cover of $x$. If $U$ is a nontrivial $\mathcal{L}$-cover of $x$ with the property that whenever $U^{\prime}$ is an $\mathcal{L}$-cover of $x$ and $U^{\prime} \ll U$

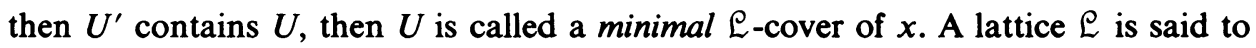
satisfy condition $\left(R_{\bigvee}\right)$ iff there exists a mapping $\rho: \mathcal{L} \rightarrow \omega$ such that, if $U$ is a minimal $\mathcal{L}$-cover of $x$ and $u \in U$, then $\rho(x)<\rho(u)$ for each $u \in U$ (here $\omega$ denotes the natural numbers). For a finite lattice, condition $\left(R_{\wedge}\right)$ can be defined as the dual of condition $\left(\mathrm{R}_{\vee}\right)$. The lattice $\mathcal{L}$ satisfies condition (W) iff for all $a, b, c, d \in L$, if $a \wedge b \leqslant c \vee d$, then either $a \leqslant c \vee d, b \leqslant c \vee d, a \wedge b<c$, or $a \wedge b<d$. The result in [3] shows that a finite lattice is sharply transferable if and only if it

Received by the editors November 13, 1979.

1980 Mathematics Subject Classification. Primary 06A20.

Key words and phrases. Lattice, transferable, ideal lattice.

${ }^{1}$ Research supported by the National Science and Engineering Research Council of Canada. 
satisfies $\left(R_{\vee}\right),\left(R_{\wedge}\right)$ and (W). We further recall that a lattice is said to satisfy condition (SF) (sectionally finite) if every principal ideal is finite. It was observed in [6] that every transferable lattice satisfies (SF).

In a note communicated to the author, $R$. Freese and $\mathbf{W}$. Lampe, making use of some unpublished ideas of J. B. Nation, gave a proof that a sublattice of a (possibly infinite) sharply transferable lattice is sharply transferable. That proof can be modified to give the following result.

THEOREM 1. If $\mathcal{L}$ satisfies $\left(\mathrm{R}_{\vee}\right)$ and $(\mathrm{SF})$ and $\mathcal{K}$ is a sublattice of $\mathcal{E}$, then $\mathcal{K}$ satisfies $\left(\mathbf{R}_{\bigvee}\right)$.

Proof. For $x \in \mathcal{L}$ such that $[x) \cap \mathcal{K} \neq \varnothing$, let $f(x)=\wedge([x) \cap \mathscr{K}) \in \mathcal{K}$. Clearly $f$ is join-preserving. Let $\rho: \mathcal{L} \rightarrow \omega$ establish $\left(R_{\vee}\right)$ for $\mathcal{L}$, and for $x \in \mathcal{K}$, define

$$
\rho^{\prime}(x)=\max \{\rho(y) \mid f(y)=x\} .
$$

If $U$ is a minimal $\mathcal{K}$-cover of $x$, choose $x_{0}$ in $\mathcal{L}$ with $f\left(x_{0}\right)=x$ and $\rho\left(x_{0}\right)=\rho^{\prime}(x)$. Then $U$ is a nontrivial $\mathcal{L}$-cover of $x_{0}$ since, if $x_{0}<u \in U$, then $x=f\left(x_{0}\right)<f(u)=$ $u$ by definition of $f$, contrary to the fact that $U$ is a nontrivial $\mathcal{K}$-cover of $x$. Since $\mathcal{L}$ satisfies (SF), there exists $U^{\prime} \ll U$ with $U^{\prime}$ a minimal $\mathcal{E}$-cover of $x_{0}$. Clearly $f\left(U^{\prime}\right) \ll U$, and $x_{0} \leqslant \bigvee U^{\prime}$, so $f\left(x_{0}\right) \leqslant f\left(\bigvee U^{\prime}\right)$. That is, $x<f\left(\bigvee U^{\prime}\right)=\bigvee f\left(U^{\prime}\right)$, so, since $U$ is a minimal $\mathscr{K}$-cover of $x$, we get $U \subseteq f\left(U^{\prime}\right)$. Thus, if $u \in U$, let $u=f\left(u^{\prime}\right), u^{\prime} \in U^{\prime}$. Then

$$
\rho^{\prime}(x)=\rho\left(x_{0}\right)<\rho\left(u^{\prime}\right) \leqslant \rho^{\prime}\left(f\left(u^{\prime}\right)\right)=\rho^{\prime}(u) . \quad \text { Q.E.D. }
$$

Let $\mathcal{L}$ be a lattice satisfying (SF). For $X \subseteq L \times \omega$ and $n \in \omega$, let $X^{(n)}=$ $\{x \mid\langle x, n\rangle \in X\}$. Call $X$ closed if and only if $\left(X^{(n+1)}\right] \subseteq X^{(n)}$ for all $n \in \omega$. If $X \subseteq L \times \omega$, let [[X]] denote the smallest closed subset of $L \times \omega$ containing $X$. Let $K$ be the set of all finite closed subsets of $L \times \omega$. The following are easily verified:

(i) if $X \subseteq L \times \omega$ is finite, then [[X]] $\in K$;

(ii) $K$ forms a lattice $\mathcal{K}$ in which, for $X$ and $Y$ in $K, X \wedge Y=X \cap Y$ and $X \vee Y=[[X \cup Y]]$

(iii) if $X \subseteq L \times \omega$, then

$$
[[X]]^{(n)}=X^{(n)} \cup\left(\cup\left(X^{(m)} \mid m>n\right)\right]
$$

(iv) $\mathscr{K}$ satisfies (SF).

LEMMA 1. $X \in K$ is join-irreducible in $\mathscr{K}$ if and only if $X=[[\{\langle x, n\rangle\}]]$ for some $x \in L$ and $n \in \omega$.

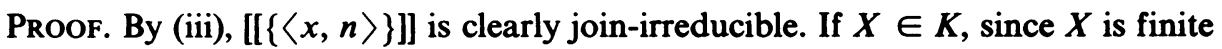
we can choose $\langle x, n\rangle \in X$ with $n$ maximal. Obviously $X-\{\langle x, n\rangle\}$ is closed, and

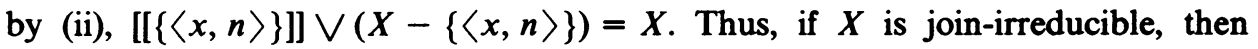
$X=[[\{\langle x, n\rangle\}]]$. Q.E.D.

Define, for $X \in K, \rho(X)=1+\max \left\{n \mid X^{(n)} \neq \varnothing\right\}$.

LEMMA 2. If $X=[[\{\langle x, n\rangle\}]]$ and $W$ is a minimal $\mathcal{K}$-cover of $X$, then $\rho(X)<$ $\rho(U)$ for all $U \in W$. 
Proof. Clearly $\rho(X)=n+1$ and $\langle x, n\rangle \in[[\cup W]]$. However since $W$ is a nontrivial $\mathscr{K}$-cover, $\langle x, n\rangle \notin \cup W$. For $U \in W$, let $U^{\prime}=[[U \cap(L \times[n+1))]]$, and put $W^{\prime}=\left\{U^{\prime} \mid U \in W\right\}$. By (iii), $\langle x, n\rangle \in\left[\left[\bigvee W^{\prime}\right]\right]$, so $X<\bigvee W^{\prime}$. But $W^{\prime} \ll W$, so $W \subseteq W^{\prime}$. Since $\varnothing \notin W$, clearly $\rho\left(U^{\prime}\right)>n+2$ for all $U \in W$. Q.E.D.

\section{Corollary. $\mathcal{K}$ satisfies $\left(\mathbf{R}_{\bigvee}\right)$.}

Proof. In view of Lemma 2 and the fact that every element of a minimal $\mathcal{K}$-cover must be join-irreducible, it suffices to redefine $\rho(X)$ to be 0 for all join-reducible $X$ in $\mathscr{K}$. Q.E.D.

LEMMA 3. $\mathcal{L}$ is embeddable into $I(\mathcal{K})$.

Proof. For $x \in L$, define $f(x)=\left\{X \in K \mid X^{(0)} \subseteq(x]\right\}$. Using (ii) and (iii) it is straightforward to check that $f(x)$ is an ideal of $\mathcal{K}$ and that $f$ is one-to-one and meet-preserving. To complete the proof it suffices to show that for $x$ and $y$ in $L$, $f(x \vee y) \subseteq f(x) \vee f(y)$. If $Z \in f(x \vee y)$ then $Z^{(0)} \subseteq(x \vee y]$, so by (iii), $Z^{(m)} \subseteq$ $(x \vee y]$ for all $m$. Since $Z$ is finite, we can choose $n$ sufficiently large that $Z \subseteq L \times(n]$. Let $X=[[\langle x, n\rangle]]$, and $Y=[[\langle y, n\rangle]]$. Then $X \in f(x), Y \in f(y)$, and $Z \subseteq(x \vee y] \times(n) \subseteq X \vee Y$. It follows that $Z \in f(x) \vee f(y)$, as required. Q.E.D.

Finally we have

THEOREM 2. Every transferable lattice satisfies $\left(\mathbf{R}_{\bigvee}\right)$.

Proof. If $\mathcal{L}$ is transferable, then $\mathcal{L}$ satisfies (SF), so the above lattice $\mathcal{K}$ satisfies (SF) and $\left(R_{\vee}\right)$, and $\mathcal{L}$ is embeddable into $I(\mathscr{K})$. By transferability, $\mathcal{L}$ is embeddable in $\mathscr{K}$, so by Theorem $1, \mathcal{L}$ satisfies $\left(R_{V}\right)$. Q.E.D.

COROLlARY. Every finite transferable lattice is sharply transferable.

Proof. It was shown in [6] that transferable lattices are semidistributive. A theorem of Day (see [1]) shows that a finite semidistributive lattice satisfies $\left(R_{V}\right)$ if and only if it satisfies $\left(R_{\wedge}\right)$. In [8] it was shown that a transferable lattice satisfies (W). In view of the characterization in [7], the proof is complete. Q.E.D.

\section{REFERENCES}

1. A. Day, Characterization of finite lattices that are bounded-homomorphic images or sublattices of free lattices, Canad. J. Math. 31 (1979), 69-78.

2. B. Jonsson and J. B. Nation, A report on sublattices of a free lattice, Universalis Algebra (Proc. Colloq. Szeged, 1975), Colloq. Math. Soc. Janos Bolyai 17 (1977), 223-257.

3. H. S. Gaskill, G. Gratzer and C. R. Platt, Sharply transferable lattices, Canad. J. Math. 27 (1975), 1246-1262.

4. H. S. Gaskill and C. R. Platt, Sharp transferability and finite sublattices of free lattices, Canad. J. Math. 27 (1975), 1036-1041. 
5. G. Gratzer, Universal algebra, Trends in Lattice Theory (Sympos. U.S. Naval Academy, Annapolis, Md., 1966), Van Nostrand Reinhold, New York, 1970, pp. 173-210.

6. G. Gratzer and C. R. Platt, Two embedding theorems for lattices, Proc. Amer. Math. Soc. 69 (1978), 21-24.

7. A A characterization of sharply transferable lattices, Canad. J. Math. 32 (1980), 145-154.

8. G. Gratzer, C. R. Platt and B. Sands, Embedding lattices into lattices of ideals, Pacific J. Math. 85 (1979), 65-75.

Department of Mathematics and Astronomy, University of Manitoba, Winnipeg, Manttoba, Canada R3T 2N2 